\title{
Ethical responsibility of researchers and subjects in drugs clinical trials
}

\begin{abstract}
The success of clinical trials depends on the ability of the researchers to respect ethical responsibilities and fulfill the related duties toward the human subjects who are direct participants in the drug clinical trials. Based on the ethical responsibility connotation, this paper analyzes the ethical responsibilities and their practice involving both researchers in the drug clinical trials and subjects as well as the related perspectives.
\end{abstract}

Keywords: drug clinical trials, ethical responsibility, researcher, subject

\author{
Volume 4 Issue I - 2018 \\ Shuangsheng Yan, Ning Liu \\ Department of Social Science, China Pharmaceutical University, \\ China
}

Correspondence: Shuangsheng Yan, Ph.D., Associate Professor. Director, the Marxism Principles of Teaching and Research Office, Department of Social Science, China Pharmaceutical University, No 639, Longmian Avenue, Jiangning District, Nanjing, P.R. China, 21 I I98, Tel +86 I330 5151 782, Email yss@cpu.edu.cn

Received: December 13, 2017| Published: January 19, 2018

\section{Introduction}

According to numerous clinical research studies on involving drugs, it is gradually found that ethical factors play an important role in such trials. Indeed, ethics is considered as one of the key factors determining the clinical trials success. With the rise of the responsible innovation research, the problem of ethical responsibility in drug clinical trials has emerged. Among the most important medical ethics we find the research conscience, responsibility, honor, reputation and herein responsibility represents an important category. ${ }^{1}$ In case of lack of ethical responsibilities toward the participants during the drug clinical trial process, such lack of ethical responsibility will not only lead to the failure of the clinical trial, but also have serious consequences and outcomes such as subject health problems and divers diseases. ${ }^{2}$ Therefore, this paper attempts to put a spotlight on the ethical responsibilities of the main stakeholders in drug clinical trials and its practice.

Ethical responsibility refers to the awareness of the moral obligations of society and the divers individuals involved in different tasks, with the same meaning as duty and mission. ${ }^{3}$ Simply because "this is an obligation", there is no need to consider other motives to act because responsibility is an ethical one. ${ }^{4}$ Prospective is the unique attribute of ethical responsibility and it emphasizes that human beings should first establish a sense of responsibility in the occurrence of their actions in order to avoid the emergence and spreading of wrong behaviors due to their lack of responsibility. On the other hand, when the act occurs, it is primordial that mankind is not only responsible for their current behavior, but also for the future consequences. ${ }^{5}$

\section{Ethical responsibility in the process of drug clinical trials}

Stakeholders of the process of drug clinical trials include researchers, subjects, sponsors, ethics committees, drug administrators, etc. ${ }^{6}$ They have a variety of ethical responsibilities in the trial. Among them, researchers and subjects are directly related to stakeholders in clinical trials. Whether the clinical trials are successful or not depends on the two factors. ${ }^{7}$ The following subsections focus on the ethical responsibilities of researchers and subjects in drug clinical trials.

\section{The ethical responsibility of the researchers}

The researchers in a clinical trial are the persons who conduct clinical trials on the subject and who are responsible for their behaviors and actions. Researchers' team usually consists of principal investigator, co-investigator, coordinating investigator, and subinvestigator. ${ }^{8}$ The Helsinki Declaration states that investigators involved in clinical trials are responsible for the life protection, health, dignity, impartiality, autonomy, privacy and personal information of the subjects. Moreover, the responsibility of the protection of a subject must be ensured by a physician with other health care professionals and not by the subjects themselves even if they signed the consent. ${ }^{9}$ However, in the actual implementation of clinical trials, there are numerous abuses that have serious consequences due to the lack of ethical responsibility seen among some research teams. For instance, in 2004, a clinical trial was conducted in Cameroon to test whether daily oral Tenofovir drug could prevent the human immunodeficiency virus (HIV)-negative patients from contracting HIV. It is understood that before conducting the clinical trials, the investigators did not make a correct full evaluation of the trial risks and the disadvantages to the subjects and they did not provide the research information in English to most of French-speaking volunteers neither. However, they allowed the participants to participate in the trial with little or no knowledge, thus it have led to the infection of five female subjects with HIV. ${ }^{10,11}$ In this case, the irresponsible behavior of the researchers could be summarized within the several aspects. Firstly, without doing an early estimation about the research risk and the possibility of the harm to the participant because of such trail. Secondly, there is no clear understanding of the subjects' personal information. Thirdly; a lack of informed consent and a lack of providing the human subjects with an explanation about the purpose of the trial the risks and other related issues. Fourthly, there was no special protection for vulnerable groups in the trial. Fifthly, the insufficiency of the post-clinical evaluation.

Most subjects are involved in clinical trials because they trust the researchers and believe in science. Therefore, the researchers must be aware of their ethical responsibilities and indeed be responsible for the life and the health of the subjects. To be more specific, their ethical responsibilities include compliance with the relevant norms of clinical trials, the use of standard operating procedures, the confirmation of 
the safety and compatibility of all trials facilities and clinical trial with the test protocols, ensure that the subjects can receive timely treatment in case of any adverse reactions resulting from the trial process and adverse reaction should be assessed, reported and published as well. In addition, to respect the subjects rights of self-protection and privacy; researchers must make sure that the subject has been informed and signed a consent corresponding to the entire experiment; ensure the accuracy and completeness of the test data and when publishing research results, both negative and positive results should be released to the public through publications or other forms.

\section{The subjects' ethical responsibility}

The clinical trials are processes of mutual trust, cooperation and mutual responsibility involving researchers and subjects. Researchers assume the responsibility of protecting the rights and well-being of the subjects. Therefore, respecting the researchers is the most basic ethical responsibility of the subjects. Specifically, the ethical responsibility of the subjects is embodied in the screening of registration forms, informed consent and participation in all aspects of the clinical trial process. ${ }^{12}$ During the registration of the screening process, the subjects ethical responsibilities includes answering truthfully the questions asked by the researchers, filling in the personal information truthfully and making the choice whether to participate in the research based on carefully weighing the advantages against the risks. For instance, investigators are required to conduct a clinical trial of new therapy for depression, but the drug addicts are not eligible to participate. ${ }^{13}$ If a non-compliant subject does not truthfully inform the investigator of his own situation, it will lead to the failure of the trial. This is not only a waste of researchers funding, time and efforts, but also a waste of qualified subjects time and effort. In the process of informed consent, the subjects' ethical responsibilities are manifested in signing the informed consent form under the condition of understanding its content and accept to voluntarily participating in the clinical trial. If subjects do not fully understand the course of participating in clinical trials, they should ask researchers to provide further detailed information. In the process of participating in clinical trials, subjects should take medication according to the regulations and always report to the investigators any symptoms and reactions occurrence following the treatment. According to the statistics of clinical trials of antiretroviral drugs for the treatment of acquired immune deficiency syndrome (AIDS), the number of participants who did not use the drug is as high as $25 \% .^{14}$ This will not only affect the clinical trials results, but may also cause direct or indirect harm to others. For instance, if a subject with tuberculosis fails to take the prescribed medication during the course of a clinical trial or fails to take certain precautions, the other subject may be infected. Furthermore, the subjects ethical responsibilities also include knowing the starting and the ending time of the clinical trial and arriving on time as agreed; notifying the investigators of the need for special medical care during the trial, always inform the investigators when the contact information (e.g. telephone number and address) change; informing the investigators if you decide to withdraw from the clinical trial and following and adhering to the clinical research withdrawing protocols.

\section{Practice path of ethical responsibility in drug clinical trials}

The purpose of exploring the ethical responsibilities of researchers and subjects in the drug clinical trials process at the theoretical level is to enable them to practically fulfill these ethical responsibilities consciously. The following will explore the practical path of ethical responsibility from the perspective of researchers and subjects respectively.

\section{Researchers should correctly fulfill their own ethical responsibilities}

Researchers, as the concrete executor of the drug clinical trials, have the arduous mission of protecting the life and health of the subjects and also promoting the well-being of the human subjects. Therefore, they must correctly fulfill their ethical responsibilities. Firstly, they need to deeply understand the requirements of clinical trials to avoid inadvertently threatening the subjects safety or affecting the integrity of the scientific research and they also need to carefully read the protocol and understand the ultimate purpose of each trial. ${ }^{15}$ Only on the basis of understanding the researchers can be able to comply with the test requirements.

Secondly, researchers have to consciously participate in training. Indeed, all researchers in clinical trials should have the opportunity to participate in international conferences or international forums on the Good Clinical Practice, the protection of human subjects and the transport of biological specimens. ${ }^{16}$ The Association of Clinical Investigators and Sponsors ${ }^{17}$ also provide researchers with reliable training and educational resources such as seminars, training and courses. Researchers should actively participate in such trainings to continuously improve their professional knowledge and professional skills.

Thirdly, strengthening communication and collaboration is among the duties of researchers as well. On one hand, researchers should strengthen communication via holding routine meetings as an important way to ensure effective communication between researchers. Researchers can use this as an opportunity to discuss the problems encountered during the process of testing and the specific required measures. The frequency of meetings can be determined based on the complexity of the clinical trial and on the number of investigators participating in that trial. On the other hand, the communication between researchers and subjects must be strengthened too. Only through communication can a researcher correctly determine whether a subject benefits from a clinical trial and make the right decision as to whether to allow him to continue participating in the trial.

Finally, researchers also need to adhere to strict self-discipline. In fact, researchers should deepen their understanding of the consequences, risks and ethical responsibilities related to the clinical trial and strictly control their own behaviors. They should not only deal with the relationship between the drug regulatory authorities, the ethics committee and the sponsors, but also deal with the relationship between the colleagues and the subjects and strive to make their behavior in line with ethical standards and norms.

\section{The subjects should properly fulfill their ethical responsibilities}

As a participant in the clinical trials of drugs, subjects who properly fulfill their ethical responsibilities must firstly correct their own motivation concerning the participation. Evidences shows that many participants in clinical trials are participating in order to obtain medical benefits or their participation is driven by economic interests rather than to promote the development of medical and health services. ${ }^{18}$ Obviously, this means the subject did not truthfully answer the investigators questions during the recruitment process or concealed the symptoms and side effects during the trial in order to remain qualified for the clinical trial, which might lead to serious 
consequences. Therefore, the subject must have a correct view of the clinical trial and be involved in clarifying his significant role and ethical responsibility.

Secondly, the subjects should comply with the test protocol. Subjects who failed to comply with the trial protocol have not only harmed themselves but also biased the test results. For instance, a woman with fertility may eventually become pregnant if she does not take effective contraception during a clinical trial. ${ }^{19}$ This does not only lead to ineffectiveness of the test results but also can drive to serious disadvantages for both woman and the health of the fetus. Subjects must recognize that adherence to the protocol is reciprocal. On one hand, individuals can benefit from the trial, such as an effective disease treatment and access to remuneration. On the other hand, it helps to test the integrity and accuracy of the data and give benefits to other patients.

Finally, the participants should cooperate with the researchers. The success of clinical trials depends largely on the cooperation between the researchers and the subjects. ${ }^{20}$ Therefore, during the process of participating in the clinical trial, the participants should actively communicate with the researcher and be able to inform the researchers their physical condition and the response after taking the drugs at any time to help the researchers correctly assess the benefits and risks, and also help them to reach the highest responsibility of their healthcare.

\section{Conclusion}

Researchers and subjects, as stakeholders are directly involved in drug clinical trials and bear different ethical responsibilities related to such trials. To achieve a high-standards clinical trial, researchers and subjects are required to adhere to these ethical responsibilities in their practice, and their clinical research process should be bordered by ethical standards and rational guidelines to enhance the human being health and boost the healthcare community achievements.

\section{Acknowledgments}

We acknowledge the Jiangsu Overseas Research \& Training Program for University Prominent Young \& Middle-aged Teachers and. We also acknowledge the Research Innovation Program Project of Graduate Students in Jiangsu Province (Project Approval Number: KYZZ16_0415).

\section{Conflict of interest}

The authors declare that there is no conflict of interest.

\section{References}

1. Cheng Maosheng. Medical Ethics. 2nd ed. China: China Medical Science Press; 2008.

2. Beauchamp T, Childress JF. Principles of biomedical ethics. New York: Oxford University Press; 2008. p. 1-432.
3. Liu Ying. The Responsibility in Ethics. Journal of Dalian University of Technology (Social Sciences). 2009;30(2):114-118.

4. Zhu Baowei. The Ethical Responsibility of Engineering Activities. Studies in Ethics. 2006;6:37-45.

5. Jonas H. The Imperative of Responsibility: In Search of Ethics for the Technological Age. USA: University of Chicago Press; 1984.

6. The Role of Purchasers and Payers in the Clinical Research Enterprise: Workshop Summary. In: Tunis S, Korn A, editors. Institute of Medicine (US) Clinical Research Roundtable. USA: National Academies Press; 2002.

7. Petryna A. Drug Development and the Ethics of the Globalized Clinical Trial. Occasional Papers. 2005;(22):1-25.

8. Apezteguía C. Reviewing Clinical Trials: A Guide for the Ethics Committee. Medicina. 2010;70(6):584-584.

9. WMA. World Medical Association Declaration of Helsinki: ethical principles for medical research involving human subjects. JAMA. 2013;310(20):2191-2194.

10. Mills E. Tenofovir trials raise ethical issues. HIV AIDS Policy Law Rev $2005 ; 10(2): 31-32$

11. SOMO briefing paper on ethics in clinical trials: Examples of unethical trials. Centre for Research on Multinational Corporations, Netherlands; 2008. 1-16p.

12. Ammassari A, Murri R, Pezzotti P, et al. Self-reported symptoms and medication side effects influence adherence to highly active antiretroviral therapy in persons with HIV infection. J Acquir Immune Defic Syndr $2001 ; 28(5): 445-449$.

13. Baer $\mathrm{AR}$, Devine $\mathrm{S}$, Beardmore $\mathrm{CD}$, et al. Clinical investigator responsibilities. J Oncol Pract. 2011;7(2):124-128.

14. Kee AN. Investigator responsibilities for clinical research studies: proper staffing can ensure an investigator is compliant. J Med Pract Manage. $2011 ; 26(4): 245-247$

15. Carmen Aldinger, Barbara E Bierer, Rebecca Li, et al. MRCT Center PostTrial Responsibilities Framework: Continued Access to Investigational Medicines-I. Guidance Document. 2017. 1-74p.

16. Rabkin J, Small E. Rights and responsibilities of research participants. Body positive magazine, USA; 2001

17. Meinert CL. Clinical Trials, Overview. Wiley Handbook of Current and Emerging Drug Therapies. USA: John Wiley \& Sons Inc; 2006.

18. Costas L, Bayas JM, Serrano B, et al. Motivations for participating in a clinical trial on an avian influenza vaccine. Trials. 2012;13:28.

19. Resnik DB, Ness E. Participants' responsibilities in clinical research. $J$ Med Ethics. 2012;38(12):746-750.

20. Chilengi R. An ethics perspective on responsibilities of investigators, sponsors and research participants. Acta Tropica. 2009;112(Suppl 1):S53-S62. 